\title{
Oncotype testing in patients undergoing intraoperative radiation for breast cancer
}

\author{
KELSEY E. LARSON ${ }^{1}$, STEPHANIE A. VALENTE ${ }^{1}$, CHIRAG SHAH $^{2}$, \\ RAHUL D. TENDULKAR ${ }^{2}$, SHEEN CHERIAN ${ }^{2}$, JAME ABRAHAM ${ }^{3}$, COURTNEY YANDA $^{1}$, \\ $\mathrm{CHAO} \mathrm{TU}^{4}$, JESSICA ECHLE ${ }^{2}$ and STEPHEN R. GROBMYER ${ }^{1}$ \\ Departments of ${ }^{1}$ Surgery, Division of Breast Services, ${ }^{2}$ Radiation Oncology, ${ }^{3}$ Medical Oncology and \\ ${ }^{4}$ Quantitative Health Science, Cleveland Clinic, Cleveland, OH 44195, USA
}

Received February 2, 2018; Accepted June 27, 2018

DOI: $10.3892 /$ mco.2018.1704

\begin{abstract}
Oncotype DX recurrence score (RS) predicts risk of distant disease recurrence, and can guide chemotherapy recommendations in hormone positive, human epidermal growth factor 2-negative, early stage breast cancer. The present study aimed to evaluate the pattern of oncotype testing, RS and adjuvant treatment in patients undergoing intraoperative radiotherapy (IORT). Single center prospective data registry was queried for patients receiving IORT between October 2011 and February 2017. Patient demographics, tumor characteristics, RS, systemic therapy and recurrence information were analyzed. A total of 150 women with mean age of 70.8 years were included. The majority had invasive ductal cancer $(60.6 \%)$ with $1.0 \mathrm{~cm}$ average tumor size and no lymph node involvement $(99 \%)$. Oncotype testing was performed in 36 patients $(24.3 \%)$. Low risk score $(<18)$ was confirmed in 19 women (53\%); intermediate risk score (18-30) in 16 women (44\%); and high risk score $(>30)$ in one woman $(3 \%)$. Patients with RS testing had significantly increased tumor sizes (1.2 vs. $1.0 \mathrm{~cm} ; \mathrm{P}<0.001)$ and were younger ( 68.5 vs. 71.3 years; $\mathrm{P}=0.02)$ compared with those not tested. A total of $4 / 150$ patients (2.6\%) received chemotherapy; two received chemotherapy based on RS testing. Based on the current selection criteria for IORT, oncotype testing rarely results in a high-risk score or utilization of chemotherapy for IORT patients. The present study supports selective use of RS testing in IORT patients and confirms that biologically low-risk patients are being selected for IORT based on current guidelines.
\end{abstract}

Correspondence to: Dr Stephen R. Grobmyer, Department of Surgery, Division of Breast Services, Cleveland Clinic, 9500 Euclid Avenue, Cleveland, OH 44195, USA

E-mail: grobmys@ccf.org

Key words: breast cancer, IORT, recurrence score

\section{Introduction}

Single dose intraoperative radiotherapy (IORT) has gained popularity in the United States since the mid-2000s (1), with low complication and in-breast recurrence rates reported in the literature (1-3). Patients selected for IORT treatment are generally low risk individuals given their older age, smaller tumors, and high rates of estrogen receptor positivity (ER+) (1). The updated American Society for Radiation Oncology (ASTRO) consensus statement on partial breast irradiation delineates specific suitable characteristics (including 50 years of age or older with $<2.5 \mathrm{~cm}$ screen detected invasive tumors of low or intermediate grade) for patients to be considered for low energy x-ray IORT treatment (3). In addition, it recommends low energy x-ray IORT should be offered as part of a prospective registry or trial.

Oncotype DX is a genomic assay which became available in the United States in 2004, with increasing use since it was first introduced (4). This assay informs risk of metastatic disease recurrence via recurrence score (RS) and thus can guide chemotherapy and antihormone therapy recommendations in select breast cancer patients. Current national guidelines including those from National Comprehensive Cancer Network (NCCN) and American Society of Clinical Oncology (ASCO) support consideration for RS testing in select patients who have small ER+, HER2 negative breast cancers $(5,6)$. RS testing can also be applied to patients with limited lymph node positive disease in select circumstances $(4,7)$.

Based on current guidelines, there exists overlap between patients who may be candidates for IORT and those who may be considered for RS testing. In addition, RS is another way to assess the risk profile of patients currently being selected for IORT. However, data on RS testing in patients receiving IORT is lacking. Therefore the purpose of this study was to evaluate the pattern of RS testing and results, subsequent adjuvant chemotherapy recommendations, and antihormone therapy use in IORT patients treated at a single institution.

\section{Materials and methods}

Patient selection. Institutional Review Board approval was in place for a prospective data registry for all patients at our center 
who undergo IORT. This registry was started in 10/2011 and is continuously updated by surgical and radiation oncology staff, as well as research coordinators. All patients at our institution have IORT administered using the Zeiss Intrabeam System after multidisciplinary evaluation. The prospective registry was queried in retrospective fashion for patients who underwent IORT from 10/2011 to 2/2017. Data collected included patient demographics, tumor characteristics, RS (if performed), adjuvant chemotherapy administration, antihormone therapy compliance, follow-up and disease recurrence. RS testing (Oncotype DX) was ordered by the patient's medical oncologist based on clinical judgment using NCCN (5) and ASCO (6) guidelines as a basis for testing recommendations.

Statistical analysis. Baseline data was summarized with counts and percentages or mean with range, where applicable. Chi-squared, Fisher's exact, and Wilcoxon rank sum tests were used to compare the patients who did and did not receive RS testing. All tests were two-tailed, and P-value $<0.05$ was considered statistically significant. R software (v3.31, 2016-0-21) was used for all analysis. The association between RS testing, scores and chemotherapy administration was also assessed, along with recurrence data.

\section{Results}

Baseline characteristics of patient population. Registry data was available for 150 women. The patients had an average age of 70.8 years (range 52-89 years) and most commonly had pure invasive ductal cancers $(91 / 150,60.6 \%)$ with $1.0 \mathrm{~cm}$ average tumor size (range $0-2.5 \mathrm{~cm}$ ) and no lymph node involvement $(149 / 150,99 \%)$. Cancers were most commonly ER+ $(149 / 150,99.3 \%)$ and $\mathrm{PR}+(132 / 150,87.3 \%)$; HER2 positivity was uncommon $(1 / 150,0.6 \%)$. Lymphovascular invasion was present in $4.6 \%(7 / 150)$ of patients. Most patients had histologic Grade $1(61 / 150,40.7 \%)$ or Grade $2(77 / 150,51.3 \%)$ disease.

Recurrence score testing and adjuvant therapy. RS testing was performed in $36 / 150$ patients $(24.3 \%)$. Low risk score $(<18)$ was confirmed in 19 women (53\% of those tested); none of these patients received chemotherapy. Intermediate risk score (18-30) was confirmed in 16 women (44\% of those tested); one patient received chemotherapy due to intermediate risk score and patient refusal to take antihormonal medication. High risk score ( $>30$ ) was confirmed in one patient (3\% of those tested); she did receive adjuvant chemotherapy as result of her score. Patients with RS testing had larger tumor size (1.0 vs. $1.2 \mathrm{~cm}, \mathrm{P}<0.001)$ and were younger (68.5 vs. 71.3 years, $\mathrm{P}=0.02$ ) compared to patients without testing. The groups were otherwise well matched with respect to patient and tumor factors. (Table I).

For the entire patient population, 4/150 patients $(2.7 \%)$ received adjuvant chemotherapy. Two individuals who had RS testing $(2 / 36,5.5 \%)$ received adjuvant chemotherapy based on $\mathrm{RS}$, as noted above. The additional patients received chemotherapy due to lymph node positive disease on final pathology $(n=1)$ and ER negative status $(n=1)$. Three patients $(2 \%)$ had subsequent external beam radiation based on final pathology: One due to close negative margin $(0.1 \mathrm{~mm})$; one due to lymph node involvement; and one due to multifocal disease in the surgical specimen.
Follow-up. Median follow-up was 1.2 years (range 1 month-4.6 years). Adjuvant antihormone treatment compliance was $85.5 \%$ at last follow-up. One patient $(0.6 \%)$ had a true local recurrence occurring in same quadrant of same breast as the original cancer. Two patients (1.3\%) had new second breast cancers in a different quadrant of the ipsilateral breast. No patients had metastatic recurrences.

\section{Discussion}

This is the largest study, to our knowledge, to evaluate RS testing utilization, results, and subsequent chemotherapy recommendations in IORT patients.

In the United States, between 10 and $39 \%$ of patients with breast cancer undergo risk stratification with RS testing $(8,9)$. Rates of testing are highest for those individuals who are borderline candidates for adjuvant chemotherapy including younger women with intermediate grade tumors and limited lymph node involvement (8). Our study demonstrates a rate of RS testing of $24.3 \%$ with younger age and smaller tumor size associated with increased likelihood of testing.

Meta-analysis indicates that on a national level, low risk score occurs in $48.8 \%$ of tested patients; intermediate risk in $39.0 \%$; and high risk in $12.2 \%$ (10). In prior studies of all breast cancer patients, high RS has been most closely associated with tumor grade and progesterone receptor status, although age, T stage, and lymphovascular invasion are also linked to increased RS $(11,12)$. The IORT patients in our study have a better RS profile compared to the national trends, and are in keeping with those documented in a prior publication on IORT patients (13). Low risk (53\%) and intermediate risk (44\%) scores are more common in IORT patients, whereas high risk score $(3 \%)$ is far less common. These results are in keeping with the patient and pathologic characteristics of the group and confirm that biologically low risk individuals are being offered IORT based on current guidelines.

Despite $24.3 \%(36 / 150)$ of IORT patients undergoing RS testing, adjuvant chemotherapy was added in only $2 / 36$ tested individuals $(5.5 \%)$ based on RS results. This is far below previous national data which indicates that adjuvant chemotherapy recommendation changes in $33 \%$ of patients after RS testing (10). Specifically, intermediate RS patients receive chemotherapy $12-80 \%$ of the time and high RS patients up to $95 \%$ of the time (14). High RS is associated with increased chemotherapy use (15). In our study, the rate of chemotherapy was $100 \%$ for high RS (one patient), but the rate of chemotherapy in intermediate risk patients was quite low $(1 / 16,6.3 \%)$. This is likely multifactorial and reflects the lower traditional clinical risk profile in the IORT patient population (1). Thus in general, RS testing is of limited clinical utility in terms of informing adjuvant chemotherapy for IORT patients with a similar profile to those in this study.

In terms of antihormonal treatment, the IORT patients in this study had a higher rate of medication compliance $(85.5 \%)$ at the time of their last follow-up compared to national averages. Nationally antihormonal medication compliance is as low as $49 \%$ (16), with highest estimates indicating 67-78\% compliance $(17,18)$. At our institution, all multidisciplinary breast cancer providers stress antihormone medication compliance at 
Table I. Comparison of patients who did and did not undergo RS testing.

\begin{tabular}{|c|c|c|c|}
\hline Characteristics & $\begin{array}{l}\text { Patients without RS } \\
\text { testing } \mathrm{n}=114(76 \%)\end{array}$ & $\begin{array}{l}\text { Patients with RS } \\
\text { testing } n=36(24 \%)\end{array}$ & P-value \\
\hline Age (years) & 71.3 & 68.5 & $0.02^{\mathrm{a}}$ \\
\hline \multicolumn{4}{|l|}{ Race } \\
\hline Caucasian & $103(90.3)$ & $32(91.4)$ & \multirow[t]{4}{*}{0.66} \\
\hline African American & $5(4.4)$ & $2(5.7)$ & \\
\hline Asian & $2(1.8)$ & 0 & \\
\hline Other & $1(0.9)$ & $1(2.9)$ & \\
\hline \multicolumn{4}{|l|}{ Tumor type } \\
\hline IDC & $68(59.6)$ & $23(63.9)$ & \multirow[t]{4}{*}{0.98} \\
\hline ILC & $4(3.5)$ & $1(2.8)$ & \\
\hline Mixed & $38(33.3)$ & $12(33.3)$ & \\
\hline Other & $4(3.5)$ & 0 & \\
\hline Tumor size $(\mathrm{cm})$ & 1.0 & 1.2 & $<0.001^{\mathrm{a}}$ \\
\hline ER-positive & $113(99.1)$ & $36(100)$ & 1.0 \\
\hline PR-positive & $99(86.8)$ & $31(86.1)$ & 0.78 \\
\hline HER2-positive & $1(0.9)$ & 0 & 1.0 \\
\hline \multicolumn{4}{|l|}{ Grade } \\
\hline 1 & $51(44.7)$ & $10(27.7)$ & \multirow[t]{3}{*}{0.15} \\
\hline 2 & $52(45.6)$ & $23(63.9)$ & \\
\hline 3 & $10(9.0)$ & $3(8.3)$ & \\
\hline
\end{tabular}

${ }^{\mathrm{a}} \mathrm{P}<0.05$, statistically significant. RS, recurrence score; ER, estrogen receptor; PR, progesterone receptor; HER2, human epidermal growth factor 2; IDC, invasive ductal carcinoma; ILC, invasive lobular carcinoma.

each visit for all ER+ patients (IORT or otherwise). This focus and increased patient communication as to the importance of these medications may contribute to the observed high compliance rate.

Overall, our study documents RS testing frequency and results in IORT patients, assesses changes in adjuvant chemotherapy treatment based on RS, and evaluates antihormonal compliance in patients who receive IORT. Currently selected IORT patients who receive RS testing are most likely to have low or intermediate risk scores; high risk scores are rare. RS testing rarely results in adjuvant chemotherapy administration. This study confirms that biologically low risk patients are being selected for IORT and supports continued selective use of RS testing in IORT patients.

\section{Acknowledgements}

Not applicable.

\section{Funding}

No funding was received.

\section{Availability of data and materials}

The datasets analyzed during this study are not publicly available as per institutional policy. Please direct inquiries to the corresponding author.

\section{Authors' contributions}

KL was involved in the study design, statistical analysis, writing of the manuscript and data interpretation. SV was involved in the study design, data collection, writing of the manuscript, data interpretation. CS, RT and SC were involved in the study design, data collection and editing of the manuscript. JA was involved in data interpretation and editing of the manuscript. CY performed statistical analysis and data collection. CT was the primary statistician. JE performed data collection. SG was involved in the study design, data interpretation, and writing and editing of the manuscript.

\section{Ethics approval and consent to participate}

Institutional Review Board approval was in place for a prospective data registry for all patients at the Cleveland Clinic who underwent IORT.

\section{Patient consent for publication}

All authors have provided consent for publication.

\section{Competing interests}

Dr Jame Abraham is a consultant speaker for Pfizer and Genentech. Dr Stephen Grobmyer has received travel support from Zeiss Meditech. Dr Chirag Shah is a scientific consultant 
for Impedimed Inc. Dr Stephanie Valente has received speaker and travel support from Zeiss Meditech.

\section{References}

1. Valente SA, Tendulkar RD, Cherian S, O'Rourke C, Greif JM, Bailey L, Uhl V, Bethke KP, Donnelly ED, Rudolph R, et al: TARGIT-R (Retrospective): North American Experience with Intraoperative Radiation Using Low-Kilovoltage X-Rays for Breast Cancer. Ann Surg Oncol 23: 2809-2815, 2016.

2. Vaidya JS, Joseph DJ, Tobias JS, Bulsara M, Wenz F, Saunders C, Alvarado M, Flyger HL, Massarut S, Eiermann W, et al: Targeted intraoperative radiotherapy versus whole breast radiotherapy for breast cancer (TARGIT-A trial): An international, prospective, randomised, non-inferiority phase 3 trial. Lancet 376: 91-102, 2010.

3. Correa C, Harris EE, Leonardi MC, Smith BD, Taghian AG, Thompson AM, White J and Harris JR: Accelerated partial breast irradiation: Executive summary for the update of an ASTRO evidence-based consensus statement. Pract Radiat Oncol 7: 73-79, 2017.

4. Roberts MC, Miller DP, Shak S and Petkov VI: Breast cancer-specific survival in patients with lymph node-positive hormone receptor-positive invasive breast cancer and Oncotype DX Recurrence Score results in the SEER database. Breast Cancer Res Treat 163: 303-310, 2017.

5. National Comprehensive Cancer Network Guidelines, B.C. NCCN guidelines version 2.2017 breast cancer. 2.2017 4.18.2017; Available from: https://www.ncen.org.

6. Harris LN, Ismaila N, McShane LM, Andre F, Collyar DE, Gonzalez-Angulo AM, Hammond EH, Kuderer NM, Liu MC, Mennel RG, et al: Use of biomarkers to guide decisions on adjuvant systemic therapy for women with early-stage invasive breast cancer: American Society of Clinical Oncology Clinical Practice Guideline. J Clin Oncol 34: 1134-1150, 2016.

7. Stemmer SM, Klang SH, Ben-Baruch N, Geffen DB, Steiner M Soussan-Gutman L, Merling S, Svedman C, Rizel S and Lieberman N: The impact of the 21-gene Recurrence Score assay on clinical decision-making in node-positive (up to 3 positive nodes) estrogen receptor-positive breast cancer patients. Breast Cancer Res Treat 140: 83-92, 2013.
8. Dinan MA, Mi X, Reed SD, Hirsch BR, Lyman GH and Curtis LH: Initial trends in the use of the 21-gene recurrence score assay for patients with breast cancer in the Medicare population, 2005-2009. JAMA Oncol 1: 158-166, 2015.

9. Lynch JA, Berse B, Coomer N and Kautter J: 21-gene recurrence score testing among Medicare beneficiaries with breast cancer in 2010-2013. Genet Med 19: 1134-1143, 2017.

10. Carlson JJ and Roth JA: The impact of the Oncotype Dx breast cancer assay in clinical practice: A systematic review and meta-analysis. Breast Cancer Res Treat 141: 13-22, 2013.

11. Orucevic A, Bell JL, McNabb AP and Heidel RE: Oncotype DX breast cancer recurrence score can be predicted with a novel nomogram using clinicopathologic data. Breast Cancer Res Treat 163: 51-61, 2017.

12. Gage MM, Rosman M, Mylander WC, Giblin E, Kim HS, Cope L, Umbricht C, Wolff AC and Tafra L: A validated model for identifying patients unlikely to benefit from the 21-gene recurrence score assay. Clin Breast Cancer 15: 467-472, 2015.

13. Oppong BA, Sen Gupta S, Gary M, Wehner P, Mete M, Zhao D, Seevaratnam S, Rudra S and Willey SC: 21-gene recurrence assay in patients receiving intraoperative radiotherapy: Are 'favorable' characteristics a surrogate for low recurrence? Gland Surg 6: 675-681, 2017

14. Enewold L, Geiger AM, Zujewski J and Harlan LC: Oncotype Dx assay and breast cancer in the United States: Usage and concordance with chemotherapy. Breast Cancer Res Treat 151: 149-156, 2015. Nguyen MT, Stessin A, Nagar H, D'Alfonso TM, Chen Z, Cigler T, Hayes MK and Shin SJ: Impact of oncotype DX recurrence score in the management of breast cancer cases. Clin Breast Cancer 14: 182-190, 2014.

15. Hershman DL, Kushi LH, Shao T, Buono D, Kershenbaum A, Tsai WY, Fehrenbacher L, Gomez SL, Miles S and Neugut AI: Early discontinuation and nonadherence to adjuvant hormonal therapy in a cohort of 8,769 early-stage breast cancer patients. J Clin Oncol 28: 4120-4128, 2010.

16. Brier MJ, Chambless DL, Gross R, Chen J and Mao JJ: Perceived barriers to treatment predict adherence to aromatase inhibitors among breast cancer survivors. Cancer 123: 169-176, 2017.

17. Henry NL, Azzouz F, Desta Z, Li L, Nguyen AT, Lemler S, Hayden J, Tarpinian K, Yakim E, Flockhart DA, et al: Predictors of aromatase inhibitor discontinuation as a result of treatment-emergent symptoms in early-stage breast cancer. J Clin Oncol 30: 936-942, 2012. 